\title{
Spherical Indentation Hardness of Shape Memory Alloys
}

Wenyi Yan*

School of Engineering and Technology, Deakin University, Geelong Victoria 3217, Australia

Qingping Sun

Department of Mechanical Engineering, The Hong Kong University of Science and Technology, Hong Kong, China

Hong-Yuan Liu

Centre for Advanced Materials Technology (CAMT), School of Aerospace, Mechanical and Mechatronic Engineering, The University of Sydney, Sydney, NSW 2006, Australia

*Corresponding author. Tel.:+61352272082; Email: wenyi.yan@deakin.edu.au

Submitted to Materials Science and Engineering A. 


\begin{abstract}
Using dimensional analysis and the finite element method, the spherical indentation hardness of SMAs is investigated. The scaling relationship between the hardness and the mechanical properties of a SMA, such as the forward transformation stress, the maximum transformation strain magnitude, has been derived. Numerical results demonstrated that the hardness increases with the indentation depth but there is no threefold relationship between the hardness and the forward transformation stress. Increasing the maximum transformation strain magnitude would reduce the hardness of the material. These research results enhance our understanding of the hardness from the spherical indentation of SMAs.
\end{abstract}

Key words: Shape memory alloys; Spherical indentation; Hardness; Finite element simulations.

\title{
1. Introduction
}

Shape memory alloys (SMAs), represented by NiTi, are well known smart materials because of their extraordinary behaviors: shape memory effect and superelastic deformation. At a relatively low temperature, these materials demonstrate shape memory effect; while at a higher temperature, these materials demonstrate superelastic phenomenon. These behaviors are due to the intrinsic thermoelastic martensitic transformation. The transformation temperatures can be designed by changing the composition. Both the shape memory effect and the superelastic deformation have been exploited to design SMA-based functional and smart structures in traditional mechanical engineering and modern 
biomechanical engineering. A representative application in biomechanical engineering is NiTi vascular stents to reinforce blood vessels by applying either shape memory behavior or superelastic mechanisms. Recently, SMA thin films have been recognized as a promising high performance material in the field of micro-electro-mechanical system (MEMS). Since SMA thin films need only a small amount of mass to heat or cool, the response time can be substantially reduced and the speed of operation increased significantly. Application of SMA thin films is considered as a core technology for actuation of some MEMS devices in extreme environments, such as radioactive, space, biological and corrosive conditions [1].

The reversible phase transformation in SMAs not only demonstrates the shape memory effect and superelastic behavior but also signals extraordinary mechanical properties when compared with ordinary metals. Among them, the superior wear-resistance of superelastic NiTi SMA has attracted attention. For examples, Richman et al. [2] discovered that NiTi alloys are much more resistant to cavitation erosion than even the best stainless steels while Jin and Wang [3] revealed that the sliding wear resistance of NiTi is better than a nitrided 38CrMoA1A alloy steel. Qian et al. [4] reported an anomalous relationship between hardness and wear properties of a superelastic NiTi alloy through their microwear tests. This high wear-resistant behaviour directly links to the low forward transformation stress and high recoverable transformation strain [5]. Based on this it is believed that superelastic NiTi SMA will have exciting new applications in tribological engineering [6].

Along with the research and application of superelastic NiTi SMA in MEMS and surface engineering, micro-indentation and nano-indentation techniques are playing a growing role 
in probing mechanical properties of SMAs. Gall et al. [7] studied the instrumented Vickers micro-indentation of single crystal NiTi SMA. Ni et al. [8] reported their indentation experimental results of a NiTi alloy. The dynamic indentation response of NiTi films sputtered on oxidized silicon substrates was examined by Ma and Komvopoulos [9]. We have decided to study the spherical indentation of superelastic NiTi SMA. The advantage in considering the spherical indentation for superelastic SMAs is that plastic deformation can be avoided if the indention force is not too high. Indentation with a spherical indenter has also been popularly applied to study other materials [10-11]. The theoretical analysis of spherical indentation can also assist in our understanding of the corresponding experimental studies. We have applied dimensional analysis and the finite element method to examine the spherical indentation response of superelastic SMAs [12]. According to our investigation, we have found that there exists a bifurcating point and a returning point in the superelastic indentation curve. Furthermore, we proved that the bifurcation force only relies on the forward transformation stress and the elastic properties of the initial austenite; and that the return force only relies on the reverse transformation stress and the elastic properties of the initial austenite. Additionally, the dimensionless functions to determine the bifurcation force and the return force were found to be identical.

In the present study, we continue investigation of the spherical indentation of NiTi SMA with a particular focus on the hardness. Traditionally, hardness is always an important result from an indentation test. We are attempting to understand what the hardness of the SMA from a spherical indentation test represents and to understand the influences of the material properties on this hardness. Dimensional analysis is applied to establish the scaling 
relationship for the spherical indentation hardness. The finite element simulations are then carried out to obtain numerical results of the established scaling relationship. The results are also compared with those from ordinary metallic materials.

\section{Material model}

The tensile stress-strain curve under a loading-unloading cycle is often used to demonstrate the deformation behaviour of a SMA. Idealized tensile stress-strain curves from a superelastic SMA and a SMA with shape memory effect are shown in Fig. 1(a) and Fig. 1(b), respectively. The tensile strain due to elastic deformation and forward transformation returns to zero completely after the removal of the tensile stress for a superelastic SMA (Fig. 1(a)). In contrast, the transformation strain resides after the removal of the tensile stress for a SMA with shape memory effect (Fig. 1(b)) (This residual strain can be recovered by increasing the temperature). Here, the processes of the forward transformation from austenite to martensite and the reverse transformation from martensite to austenite are simplified as perfect, i.e., the forward transformation stress, $\sigma_{f}$, and the reverse transformation stress, $\sigma_{r}$, are respectively constant during the forward and reverse transformation processes. The forward transformation process occurs in the loading process. The reverse transformation process only occurs in the unloading process for a superelastic SMA but it will not happen in the unloading process for a SMA with the shape memory effect. As illustrated in Fig. 1, $\varepsilon^{t r}$ is the maximum transformation strain in uniaxial tension or the maximum magnitude of the transformation strain, $E_{a}$ is the elastic modulus of the austenite, and $E_{m}$, the elastic modulus of the martensite. Additionally, $v_{a}$ and $v_{\mathrm{m}}$ state for the elastic Poisson's ratios of the austenite and the martensite, respectively. The values of 
all these material variables can be determined from experiments. During a martenstic transformation process, the volume of the material will also change. However, the transformation volume strain is normally much smaller than the transformation shear strain components. For example, it equals $-0.37 \%$ for $\mathrm{CuAlNi}$ SMA and about $-0.39 \%$ for NiTi SMA $[13,14]$. Therefore, the transformation volume strain is neglected in our analysis.

Fig. 1 shows a one-dimensional model to describe the superelastic phenomenon. For the theoretical study of the indentation test on a superelastic alloy such as NiTi, a threedimensional superelastic transformation model is required. Research on the constitutive model for the transformation behaviour in SMA has been conducted for decades. Numerous mathematical models can be found in literatures [15-18]. To avoid the complexity of tracking the detailed evolution of the material microstructure during the phase transformations, a phenomenological model is adopted in the present investigation $[19,20]$. This phenomenological model can describe the macroscopic superelastic and shape memory deformation behavior of a SMA due to microstructural phase transformation or invariant reorientation. It is well verified and implemented in the finite element package ABAQUS [21].

\section{Spherical Indentation Analysis}

In the present investigation of the spherical indentation test, the plastic deformation is excluded, which can be easily realized in a spherical indentation test by choosing a low indentation force. In this way, we can focus our investigation on the indentation curve due to transformation and reveal the relationships between indentation curve and the 
transformation behavior of the SMA. The coupling behavior between transformation deformation and plastic deformation is quite complicated and unclear, which is one of the focuses of our experimental studies. Some experimental observations and simple theoretical modeling can be found respectively in [22, 23].

The spherical indenter tip is commercially available with different tip radii. In the case of a SMA with superelasticity, a typical superelastic indentation curve from our experimental test is shown in Fig. 2. In the test, the maximum indentation force is just over $10000 \mu \mathrm{N}$ and the corresponding indentation depth is about $120 \mathrm{~nm}$. As can be seen, the indentation curve demonstrates superelasticity as in uniaxial tension. The material transformed to martensite in the loading process can transform back to its original austenite in the unloading process. Correspondingly, the indention displacement can recover completely, which forms a closed superelastic loop.

The indenter tip is normally made of diamond. The Young's modulus of diamond is much higher than that of SMA. Therefore, we assume that the diamond is rigid in our theoretical analysis. An indentation into a superelastic SMA is illustrated in Fig. 3. As the indentation depth and the size of the indentation area are much smaller than the size of the SMA specimen, the specimen is treated as a semi-infinite body. Due to the deformation of the material, the indenter displacement, i.e., the indentation depth, $h$, is different from the vertical displacement, $h_{c}$, along which contact is made. If $h$ is smaller than $h_{c}$, then "pileup" of the surface profiles occurs, as illustrated in Fig.3. On the contrary, if $h$ is larger than $h_{c}$, then this phenomenon is called "sink-in". The degree of "sink-in" or "pile-up" depends 
on the plastic yield stress and the level of the strain-hardening behavior for ordinary elasticplastic materials [24]. The variable $a_{c}$ in Fig. 3 represents the radius of the contact area.

The finite element method is utilized to simulate the indentation of a superelastic SMA. In our numerical study, the axisymmetric condition is applied. The influence of the friction between the indenter and the specimen is considered small and neglected. Figure 4 illustrates the finite element model. The size of the entire model is much larger than the radius of the indentation tip and the size of the transformation area beneath the contact zone. The bottom of the model is, therefore, constrained in both the radial and axial directions. As demonstrated in Fig. 4(b), a very fine mesh with the shortest element side of $0.1 \mu \mathrm{m}$ is employed in the transformation zone beneath the indenter tip to ensure the accuracy of the numerical results. In total, the model contains 2940 four-noded axisymmetric elements. Testing results from elastic contact are verified by means of Hertz contact theory.

Dimensional analysis has been proved to be a powerful method to study the indentation on ordinary materials [25-27]. We have applied this method to analyze the spherical indentation response of superelastic SMAs [12]. Some of the results are briefly summarized below.

Generally, the indentation force $F$ depends on the superelastic properties of the material, which can be quantified by the parameters $\sigma_{f}, \sigma_{r}, E_{a}, E_{m}, \varepsilon^{t r}, v_{a}, v_{\mathrm{m}}$, the indentation depth $h$ and the indenter radius $R$. According to Buckingham $\Pi$ theorem for dimensionless analysis, we choose $E_{a}$ and $R$ as the primary quantities in our problem with two fundamental 
dimensional units, length and force. The dimensionless scaling relationship for the indentation force during the loading process is

$$
\frac{F}{R^{2} E_{a}}=\Pi_{L}\left(\frac{\sigma_{f}}{E_{a}}, \frac{E_{m}}{E_{a}}, \varepsilon^{t r}, v_{a}, v_{m}, \frac{h}{R}\right) .
$$

During unloading, material starts to transform back from martensite to austenite. The reverse transformation stress $\sigma_{r}$ now affects the indentation unloading force $F$.

Additionally, the unloading begins after the indenter reaches the maximum displacement $h_{m}$. Therefore, the indentation unloading force is also a function of the maximum indentation depth. The scaling function for the indentation unloading force can be derived as

$$
\frac{F}{R^{2} E_{a}}=\Pi_{U}\left(\frac{\sigma_{f}}{E_{a}}, \frac{\sigma_{r}}{E_{a}}, \frac{E_{m}}{E_{a}}, \varepsilon^{t r}, v_{a}, v_{m}, \frac{h_{m}}{R}, \frac{h}{R}\right) .
$$

As shown in Fig. 5, if we compare a superelastic indentation curve from a superelastic SMA with an elastic indentation curve from an virtual material with the same elastic properties as the austenite of the SMA, then we can find that the superelastic indentation curve bifurcates from the elastic indentation curve at the bifurcating point and it returns back to the elastic indentation curve at the returning point during the unloading process. Referring to Fig. 5(b), the indentation force at the bifurcating point is named as the bifurcation force $F_{b}$ and the indentation force at the returning force as the return force $F_{r}$. It can be proved that the bifurcation force $F_{b}$ is only a function of the Young's modulus $E_{a}$, Poisson's ratio $v_{a}$ of the austenite and the forward transformation stress $\sigma_{f}$, while the return force $F_{r}$ only relies on the Young's modulus $E_{a}$, Poisson's ratio $v_{a}$ of the austenite and the reverse transformation stress $\sigma_{r}[12]$, i.e., 


$$
\frac{F_{b}}{R^{2} E_{a}}=\Pi_{b}\left(\frac{\sigma_{f}}{E_{a}}, v_{a}\right) \quad \text { and } \quad \frac{F_{r}}{R^{2} E_{a}}=\Pi_{r}\left(\frac{\sigma_{r}}{E_{a}}, v_{a}\right)
$$

Furthermore, it has been shown that the two scaling functions $\Pi_{b}$ and $\Pi_{r}$ are identical and therefore,

$$
\Pi_{b}\left(\frac{\sigma}{E_{a}}, v_{a}\right)=\Pi_{r}\left(\frac{\sigma}{E_{a}}, v_{a}\right)
$$

The above dimensionless relationships (3) can be determined theoretically. Based on this conclusion, a method to measure the forward transformation stress and reverse transformation stress from a spherical indentation test has been proposed [28].

\section{Spherical Indentation Hardness}

Traditionally, the measurement of the hardness of a material is the major purpose of indentation tests with different indenter tips [29]. The hardness $H$ is normally defined as the mean pressure the material will support under load [30], i.e.,

$$
H=\frac{F_{m}}{A_{c}}
$$

where $F_{m}$ is the maximum indentation force. $A_{c}$, named as the contact area, is the projected area of contact under the maximum indentation force $F_{m}$. As illustrated in Fig. 2, the contact area $A_{c}$ is equal to $\pi a_{c}^{2}$ for the current spherical indenter.

Based on the definition of Eq. (5), the hardness $H$ corresponds to the maximum indentation force. As reverse transformation does not occur during a loading process, the hardness $H$ 
does not depends on the reverse transformation process. That is, the hardness analysis is suitable for both superelastic SMA and a SMA with the shape memory effect. Since the hardness has nothing to do with the reverse transformation stress, $\sigma_{r}$, the dimensionless function to determine the hardness $H$ can be expressed as

$$
\frac{H}{E_{a}}=\Pi_{H}\left(\frac{\sigma_{f}}{E_{a}}, \frac{E_{m}}{E_{a}}, \varepsilon^{t r}, v_{a}, v_{m}, \frac{h_{m}}{R}\right),
$$

where $h_{m}$ is the maximum indentation depth.

Finite element calculations were performed to exam the scaling relationship Eq. (6). Figure 6 shows the influence of the normalized forward transformation stress $\sigma_{f} / E_{a}$ on the normalized hardness $H / E_{a}$ with different ratios of Young's moduli $E_{m} / E_{a}$. The maximum magnitude of the transformation strain $\varepsilon^{t r}$ is chosen as $4 \%$, which represents a typical value for a NiTi SMA. The normalized maximum indentation depth $h_{m} / R$ in Fig. 6(a) is 0.02 and in Fig. 6(b) is 0.04 . Figure 6 indicates that the normalized hardness increases with the normalized forward transformation stress. In a certain sense, the forward transformation stress for SMAs is similar to the yield stress for ordinary metals. Increasing the value of the forward transformation stress would increase the stiffness of the structure and therefore, the indentation hardness. Figures 6(a) and 6(b) also indicates that the higher the Young's modulus of the martensite, the larger the hardness value will be, especially in the case of the larger maximum indentation depth of $h_{m} / R=0.04$.

Figure 7 shows the influence of the maximum magnitude of the transformation strain $\varepsilon^{\text {tr }}$ on the normalized hardness $H / E_{a}$ with different ratios of Young's moduli $E_{m} / E_{a}$. In all the 
cases, the normalized forward transformation stress $\sigma_{f} / E_{a}$ is chosen as 0.012 . The normalized maximum indentation depth $h_{m} / R$ is 0.02 in Fig. 7(a) and 0.04 in Fig. 7(b), respectively. With low values of $\varepsilon^{t r}$ and $E_{m} / E_{a}$, numerical instability can occurs. That is the reason for the lack of some data points in Figures 7(a) and 7(b). Both figures indicate that the normalized hardness decreases with an increase in the maximum magnitude of the transformation strain, with the exception for $h_{m} / R=0.02$ when $\varepsilon^{t r}$ exceeds $4 \%$ as shown in Fig. 7(a). This exception is because the transformation strain in the transformed zone under the indenter cannot increase further for the given smaller maximum indentation depth, even though the allowable transformation strain is larger than $4 \%$. Sufficient indentation depth is required to reflect the effect of the maximum transformation strain on the indentation hardness as demonstrated in Fig. 7(b). A large value of the maximum transformation strain magnitude suggests a large deformation is allowed, which then leads to a low value of indentation hardness, as shown in Fig. 7. A large value of the maximum transformation strain magnitude can lead not only to a low value of average contact pressure (hardness), but also a low value of the maximum contact pressure [5]. The maximum contact pressure is directly linked to the wear state of a ductile material. The wear rate increases with the maximum contact pressure under a given load [31]. Therefore, if a measured low hardness of SMA is due to a large transformation strain, then it would result in a high wear resistance, which can explain the recent observed anomalous relationship between hardness and wear properties of a superelastic NiTi SMA [4]. 
The influence of the normalized maximum indentation depth $h_{m} / R$ on the normalized hardness $H / E_{a}$ with different ratios of the Young's moduli $E_{m} / E_{a}$ is shown in Fig. 8. In all the cases for SMAs, the normalized forward transformation stress $\sigma_{f} / E_{a}$ is chosen as 0.012 and the maximum transformation strain magnitude $\varepsilon^{\text {tr }}$ is $4 \%$. Figure 8 clearly indicates that the indentation hardness of the SMA increases with the maximum indentation depth. Therefore, the spherical hardness defined by Eq. (5) cannot be treated as a material property for SMAs. These results from SMAs are compared with those from the spherical indentation of an ordinary elastic-perfectly plastic material with a yield strength $\sigma_{Y}=600 \mathrm{MPa}$ and Young's modulus $E=200 \mathrm{GPa}$, as shown in Fig. 8. For this ordinary metal, the spherical hardness does not change with the indentation depth. We believe that the effect of indentation depth on the hardness of SMAs is related to the constitutive behaviour of this special material. For SMAs, the transformation strain is limited. As illustrated in Fig. 1, the maximum transformation strain magnitude is limited. After the forward transformation finishes at a material point, if there is a further increase in the external loading, i.e., the indentation depth, the transformed martensite will undergo pure elastic deformation and, therefore, the structure will be stiffer, which results higher hardness. In contrast, for ordinary metals, ideally, there is no limit for plastic flow. Increasing the indentation depth will also proportionally increase the projected contact area. This can explain why the spherical hardness of elastic-plastic materials does not change with the indentation depth.

The ratio of hardness to yield strength is an interesting variable for ordinary metals and is around 3.0 when significant plastic deformation occurs in the contact zone [29, 32]. Here 
for the spherical indentation of SMAs, the functional relationship of the ratio $H / \sigma_{f}$ can be expressed from Eq. (6) as follows

$$
\frac{H}{\sigma_{f}}=\frac{E_{a}}{\sigma_{f}} \Pi_{H}\left(\frac{\sigma_{f}}{E_{a}}, \frac{E_{m}}{E_{a}}, \varepsilon^{t r}, v_{a}, v_{m}, \frac{h_{m}}{R}\right) .
$$

The numerical results for this ratio versus $\sigma_{f} / E_{a}$ are shown in Fig. 9. In all the cases, $\varepsilon^{t r}=4 \%$ and $h_{m} / R=0.02$. Figure 9 reveals that the ratio $H / \sigma_{f}$ decreases with an increase in the normalized forward transformation stress $\sigma_{f} / E_{a}$. In the reasonable range of $0.004 \leq \sigma_{f} / E_{a} \leq 0.016, H / \sigma_{f}$ varies from about 5.9 to 2.1. The Young's modulus of the martensite can also affect this ratio slightly.

Figure 10 further demonstrates the relationship between the ratio $H / \sigma_{f}$ and the normalized maximum indentation depth $h_{m} / R$. It clearly indicates that the ratio $H / \sigma_{f}$ evolves from about 2.6 to 5.0 when $h_{m} / R$ increases from 0.01 to 0.05 . These results are also compared with those from the same spherical indentation of an ordinary elastic-perfectly plastic material with a yield strength $\sigma_{Y}=600 \mathrm{MPa}$ and Young's modulus $E=200 \mathrm{GPa}$. The difference is very obvious for this ordinary metal, and the ratio $H / \sigma_{Y}$ slightly varies from 2.9 to 2.8 when the normalized indentation depth changes from 0.01 to 0.05. These results indicate that the spherical hardness of SMAs due to phase transformation cannot be used as a material constant to give a measure of the forward phase transformation stress. For the purpose of calibrating the transformation stress, it is necessary to examine the indentation curve [28]. 


\section{Conclusions}

Dimensional analysis and the finite element approach were employed to study the spherical indentation hardness of SMAs. The reverse transformation stress doest not affect the hardness of a superelastic SMA. The scaling relationship between the hardness and other material parameters has been established through the dimensional analysis. The influences of all the parameters on the spherical indentation hardness have been examined using the numerical results. The major conclusions from the present investigation are as follows:

1). The hardness increases with the forward transformation stress.

2). A higher Young's modulus of the martensite will lead to a larger hardness.

3). The hardness decreases with an increase in the maximum transformation strain magnitude.

4). The indentation hardness increases with the maximum indentation depth. Therefore, the indentation hardness cannot be treated as a material property of SMAs.

5). The ratio of the hardness to the forward transformation stress is not a constant. It decreases with an increase in the forward transformation stress and increases with the maximum indentation depth. The hardness cannot be used as a measure of the forward transformation stress.

\section{Acknowledgements}

This work was financially supported by the Australian Research Council and the Research Grants Council of Hong Kong. The numerical calculations were carried out at the National Facility of the Australian Partnership for Advanced Computing through an award under the Merit Allocation Scheme to WY. 


\section{References}

[1] Y. Y. Fu, H. Du., W. Huang, S. Zhang, M. Hu, Sensors and Actuators A 112 (2004), 395.

[2] R. H. Richman, A. S. Rao, D. Kung, Wear 181-183 (1995), 80.

[3] J. Jin, H. Wang, Acta Metallurgica Sinica 1 (1988), 76.

[4] L. Qian, X. Xiao, Q. Sun, T. Yu, Applied Physics Letters 84 (2004), 1076.

[5] W. Yan, submitted to Materials Science and Engineering A (2006).

[6] D. Y. Li, Smart Materials and Structures 9 (2000), 717.

[7] K. Gall, K. Juntunen, H. J. Maier, H. Sehitoglu, Y. I. Chumlyakov, Acta Materialia 49 (2001), 3205.

[8] W. Ni, Y.-T. Cheng, D. S. Grummon, Applied Physics Letters 82 (2003), 2811.

[9] X.-G. Ma, K. Komvopoulos, Applied Physics Letters 84 (2004), 4274.

[10] J. Alcala, A. E. Giannakopoulos, S. Suresh, Journal of Materials Research 13 (1998), 1390.

[11] I. Zarudi, L. C. Zhang, M. V. Swain, Applied Physics Letters 82 (2003), 1027.

[12] W. Yan, Q. Sun, X.-Q. Feng, L. Qian, to appear in International Journal of Solids and Structures (2006).

[13] D. -N. Fang, W. Lu, W. Yan, T. Inoue, K.-C. Hwang, Acta Materialia. 47 (1998), 269.

[14] R. L. Holtz, K. Sadananda, M. A. Imam, International Journal of Fatigue 21 (1999), s137.

[15] C. Liang, C. A. Rogers, Journal of Intelligent Material Systems and Structures 1 (1990), 207. 
[16] L. C. Brinson, R. Lammering, International Journal of Solids and Structures 23 (1993), 3261.

[17] Q.-P. Sun, K.-C. Hwang, Journal of the Mechanics and Physics of Solids 41 (1993), 1.

[18] W. Yan, Q.-P. Sun, K.-C. Hwang, Science in China (Series A) 41(1998), 878.

[19] F. Auricchio, R. L. Taylor, Computer Methods in Applied Mechanics and Engineering 143(1997), 175.

[20] F. Auricchio, R. L. Taylor, J. Lubliner, Computer Methods in Applied Mechanics and Engineering 146(1997), 281.

[21] ABAQUS version 6.4 (2004), Hibbitt, Karlsson and Sorensen, Providence, RI.

[22] A. L. McKelvey, R. O. Ritchie, Metallurgical and Materials Transactions A 32 (2001), 731.

[23] W. Yan, C. H. Wang, X. P. Zhang, Y.-W. Mai, Materials Science and Engineering A 354 (2003), 146.

[24] A. E. Giannakopoulos, S. Suresh, Scripta Materialia 40 (1999), 1191.

[25] Y.-T. Cheng, C. M. Cheng, International Journal of Solids and Structures 36 (1999), 1231.

[26] M. Dao, N. Chollacoop, K. J. Van Vliet, T. A. Venkatesh, S. Suresh, Acta Materialia 49 (2001), 3899 .

[27] K. Tunvisut, N. P. O’Dowd, E. P. Busso, International Journal of Solids and Structures 38 (2001), 335.

[28] W. Yan, Q. Sun, X.-Q. Feng, L. Qian, submitted to Applied Physics Letters (2006).

[29] D. Tabor, Philosophical Magazine A 74 (1996), 1207.

[30] W. C. Oliver, G. P. Pharr, Journal of Materials Research 7 (1992), 1564. 
[31] W. Yan, E. P. Busso, N. P. O'Dowd Proceedings of Royal Society London Series A 456 (2000), 2387.

[32] K. L. Johnson, Contact Mechanics, Cambridge University Press, London, 1985. 


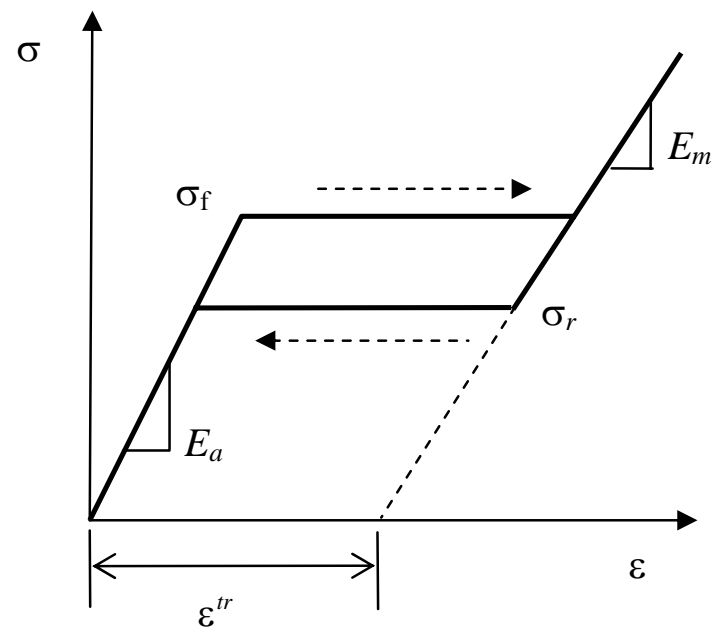

(a)

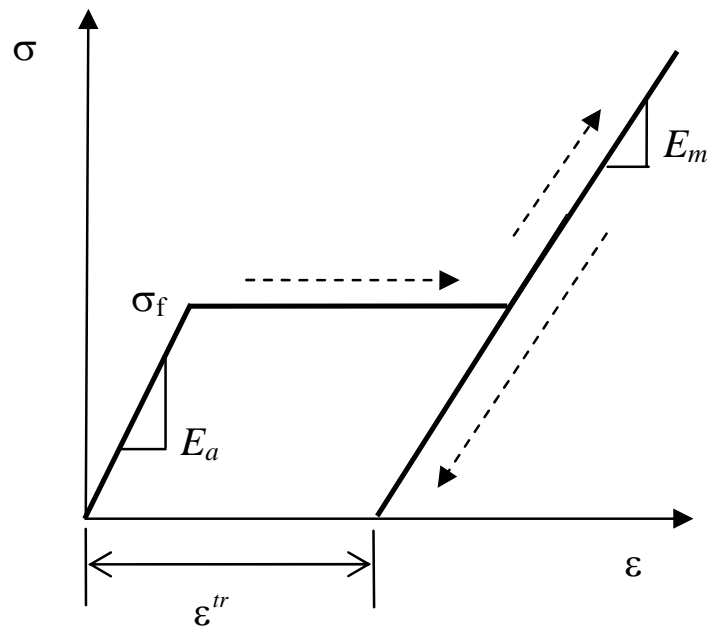

(b)

Fig. 1. Illustration of idealized stress-strain curves under a loading and unloading cycle in a uniaxial tension: (a) superelastic SMAs and (b) SMAs with shape memory effect. 


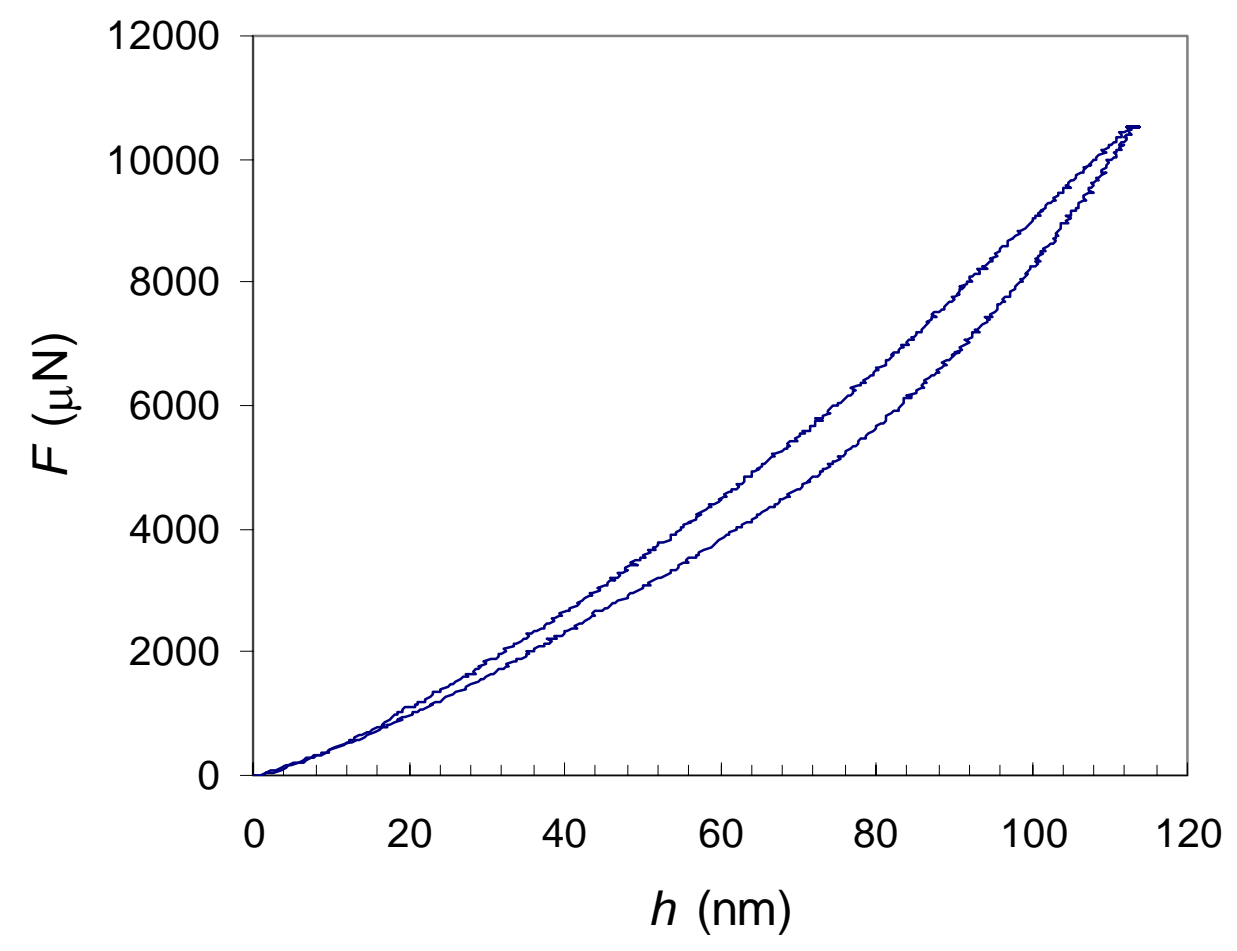

Fig. 2. A typical experimental superelastic indentation curve from an micro-indentation test of a superelastic NiTi.

Submitted to Materials Science and Engineering A. 


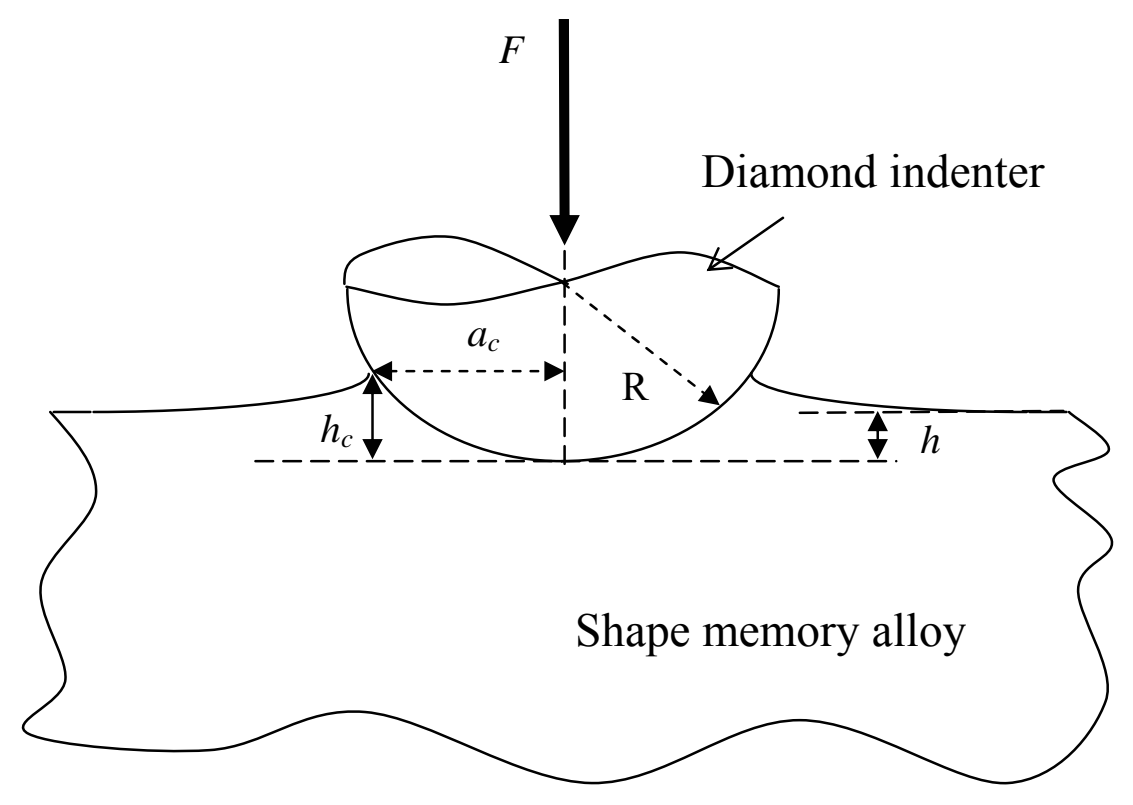

Fig. 3. Illustration of a spherical indentation test of a superelastic SMA.

Submitted to Materials Science and Engineering A. 


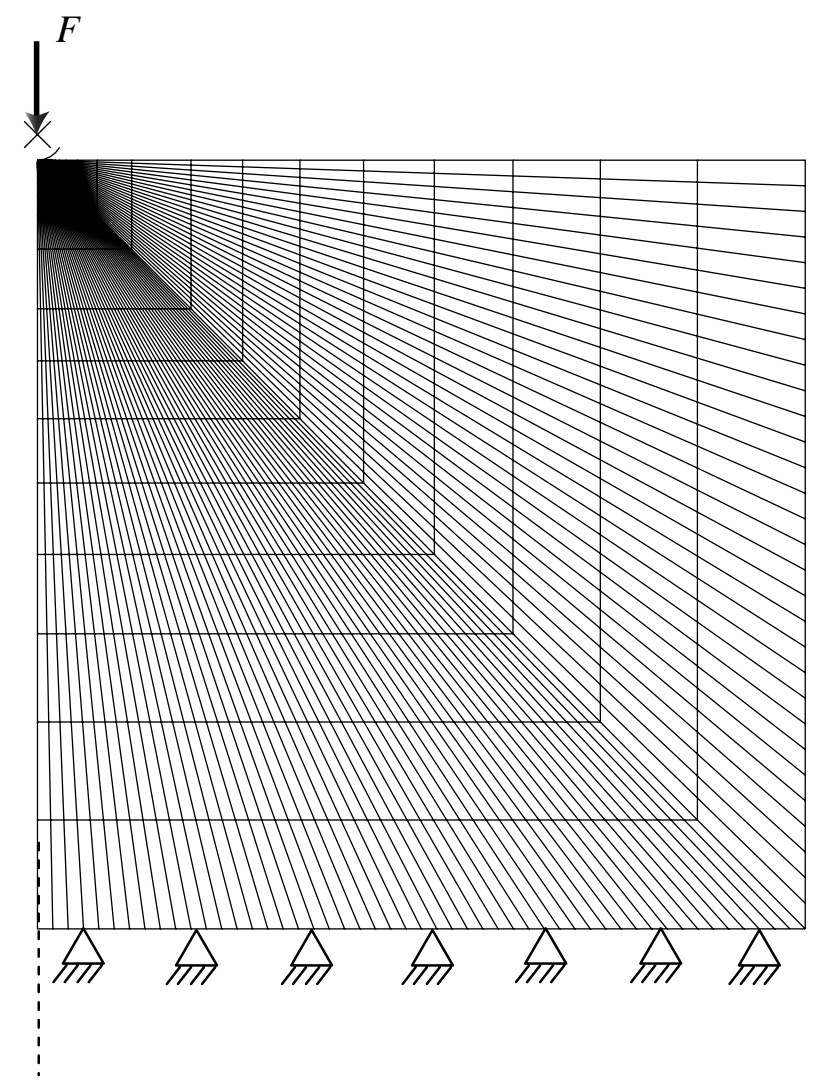

Axisymmetric axis

Fig. 4(a)

Submitted to Materials Science and Engineering A. 


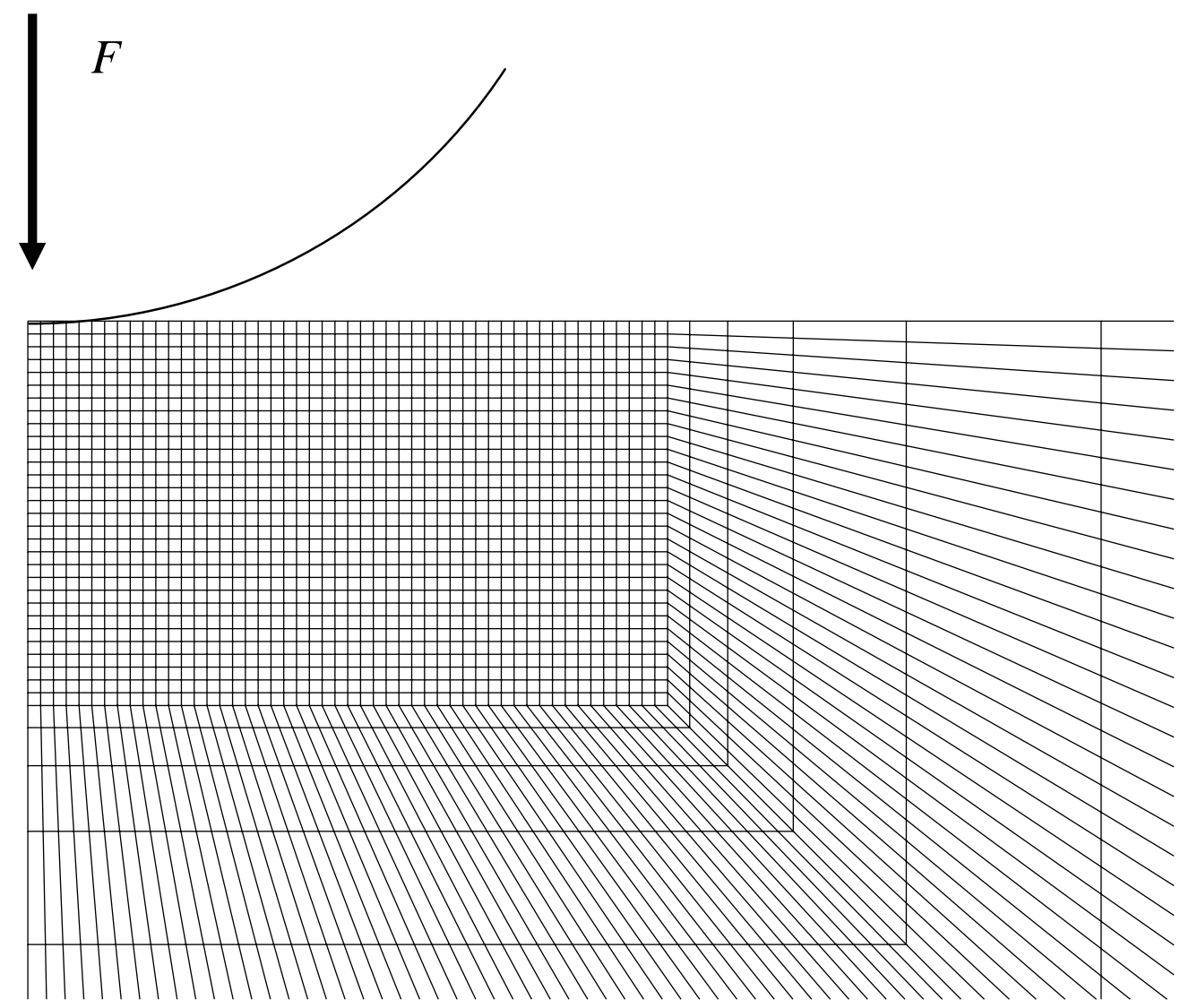

Fig. 4(b)

Fig. 4. Axisymmetric finite element model to simulate indentation test with a rigid spherical indenter: (a) entire finite element mesh and boundary conditions; (b) fine mesh near the intender tip.

Submitted to Materials Science and Engineering A. 


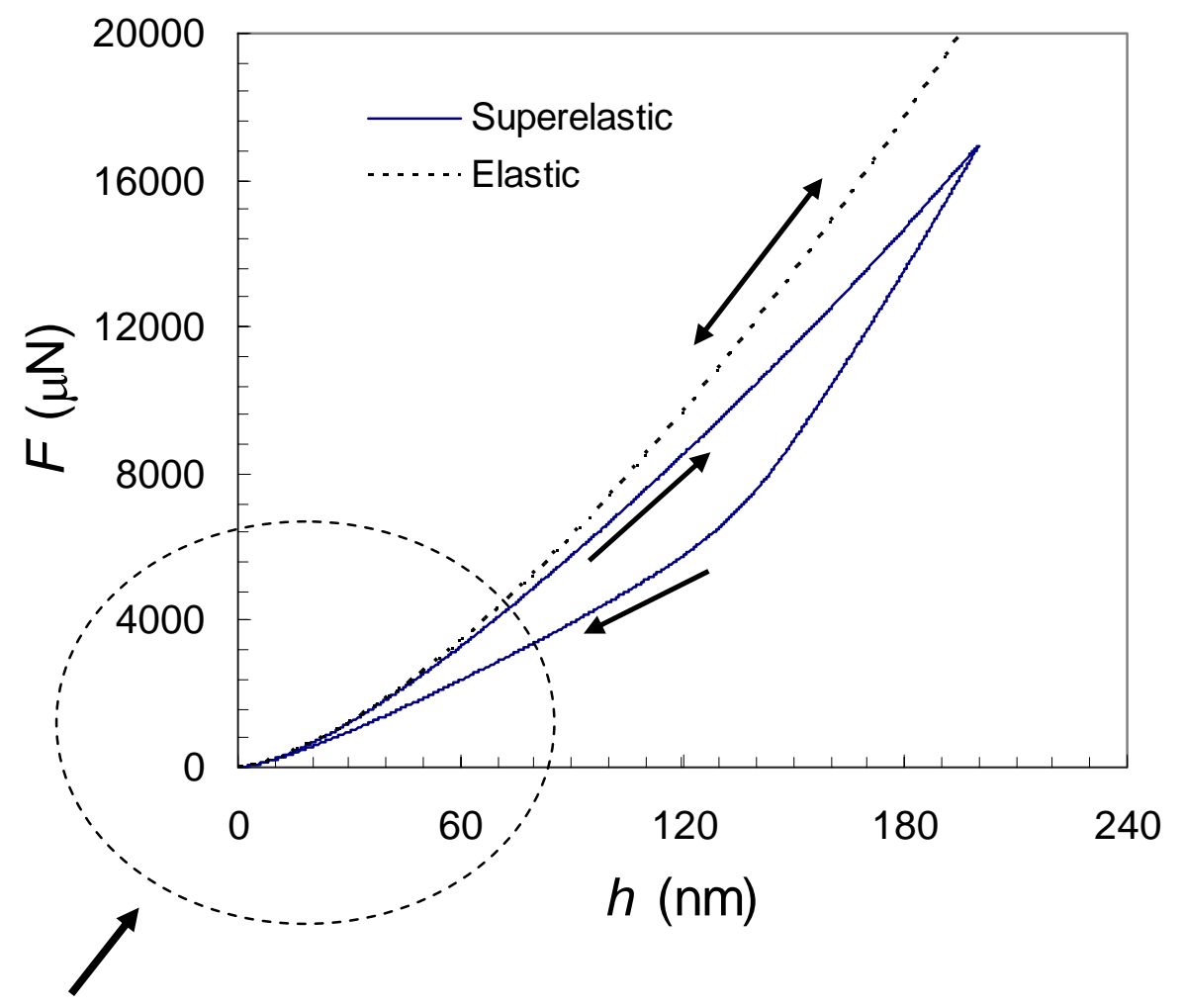

See Fig. 5(b)

Fig. 5(a). Comparison between an elastic indentation curve and a superelastic indentation curve with same elastic constants for the austenite.

Submitted to Materials Science and Engineering A. 


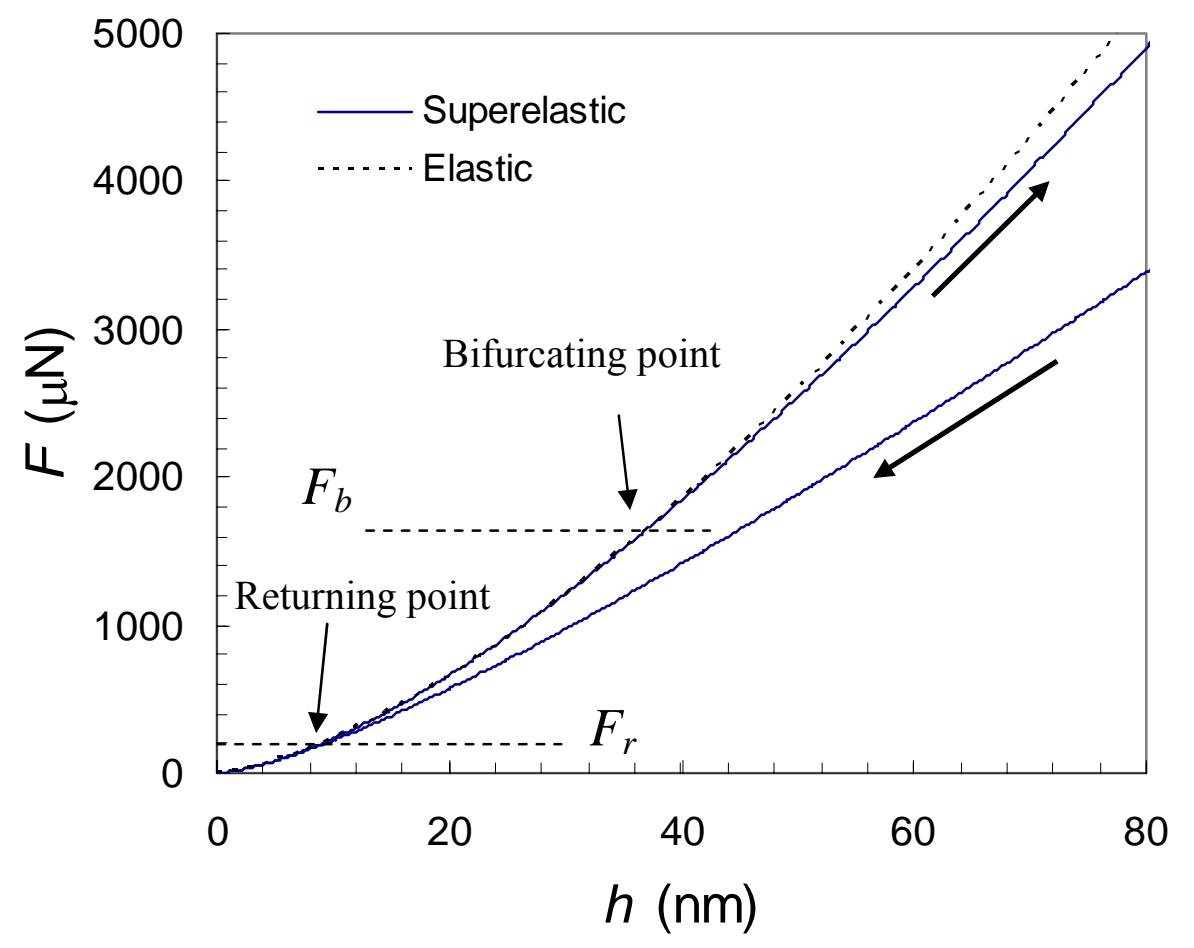

Fig. 5(b). The bifurcating point, the returning point and their corresponding indentation forces $F_{b}$ and $F_{r}$. 


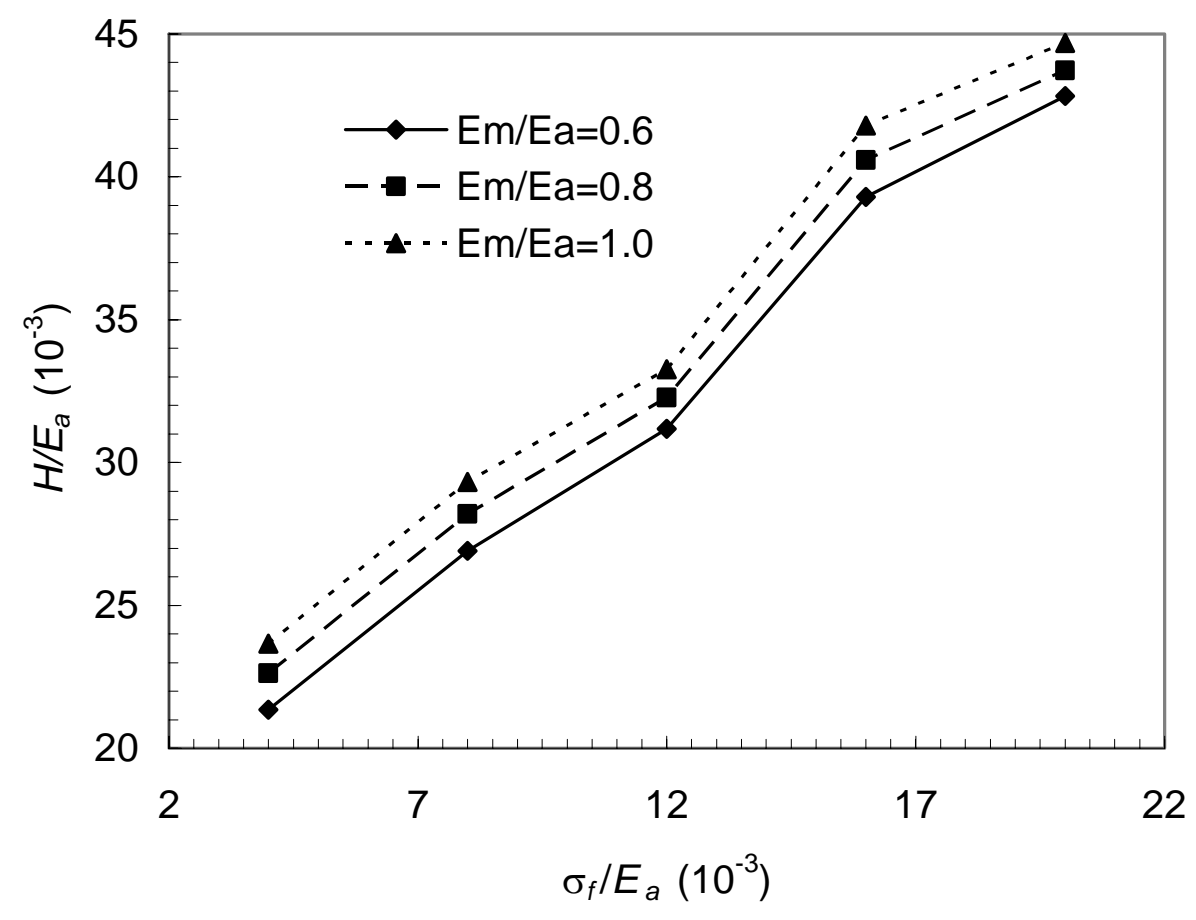

Fig. 6(a)

Submitted to Materials Science and Engineering A. 


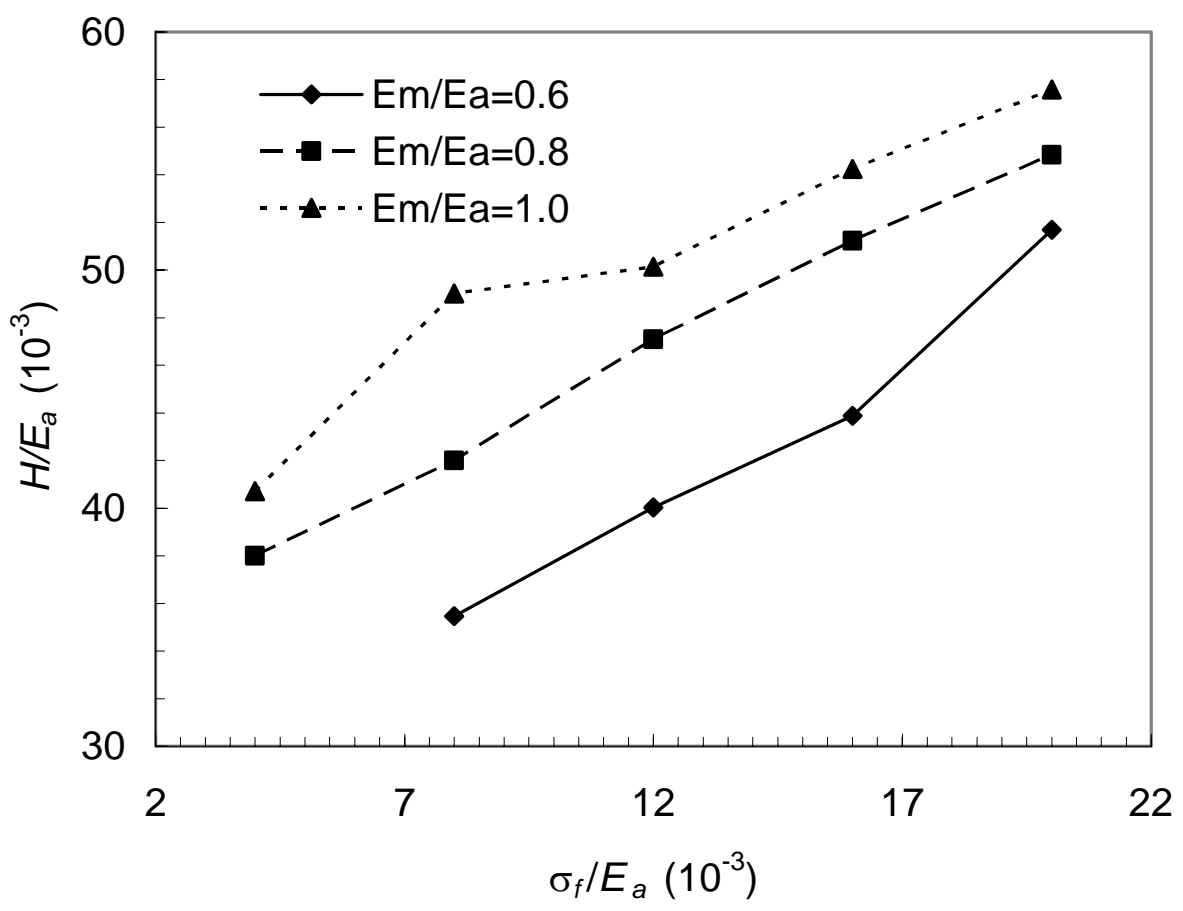

Fig. 6(b)

Fig. 6. Influence of the normalized forward transformation stress $\sigma_{f} / E_{a}$ on the normalized hardness $H / E_{a}$ for different ratios of $E_{m} / E_{a}$ with fixed $\varepsilon^{t r}$ of $4 \%$ and (a) $h_{m} / R=0.02$; (b) $h_{m} / R=0.04$. 


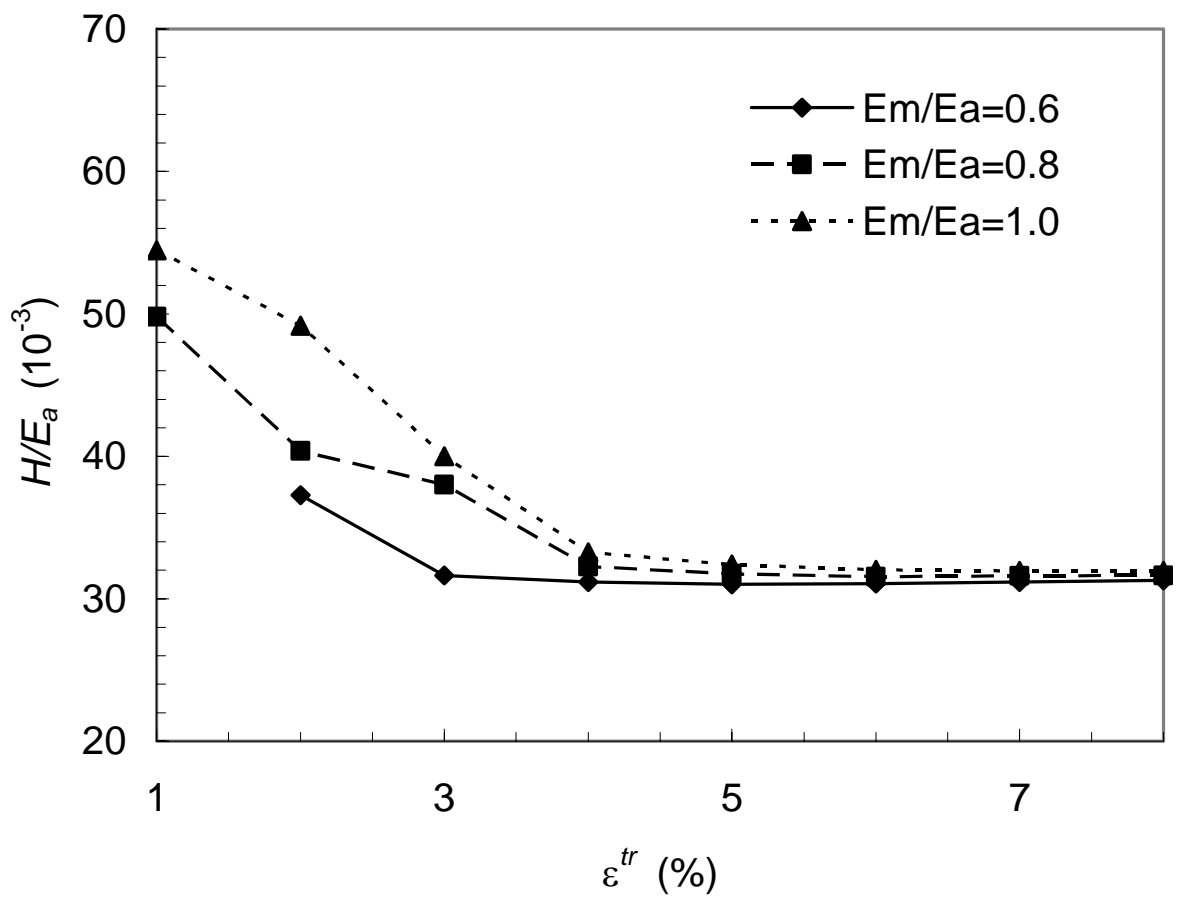

Fig. 7(a) 


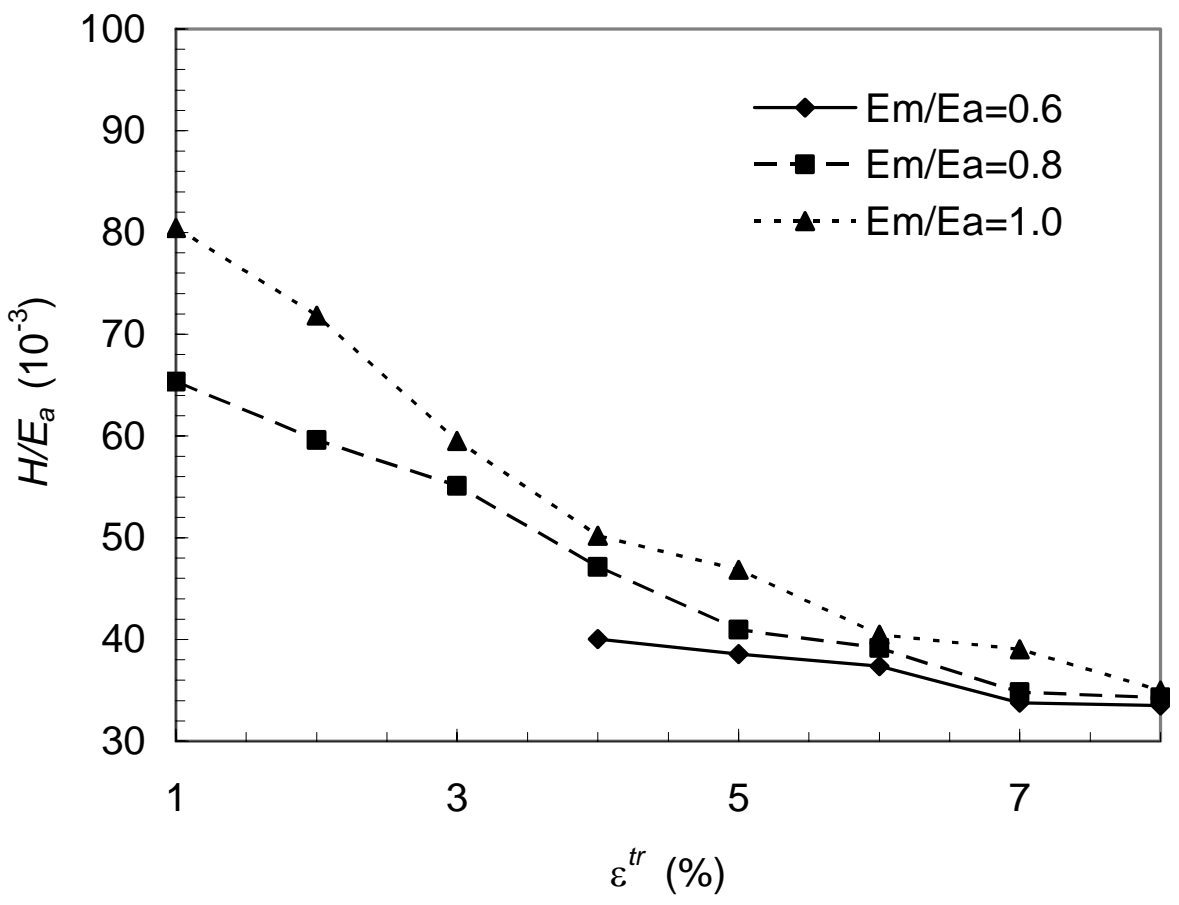

Fig. 7(b)

Fig. 7. Influence of the maximum transformation strain magnitude $\varepsilon^{\text {tr }}$ on the normalized hardness $H / E_{a}$ for different ratios of $E_{m} / E_{a}$ with fixed $\sigma_{f} / E_{a}$ of 0.012 and (a) $h_{m} / R=0.02 ;$ (b) $h_{m} / R=0.04$. 


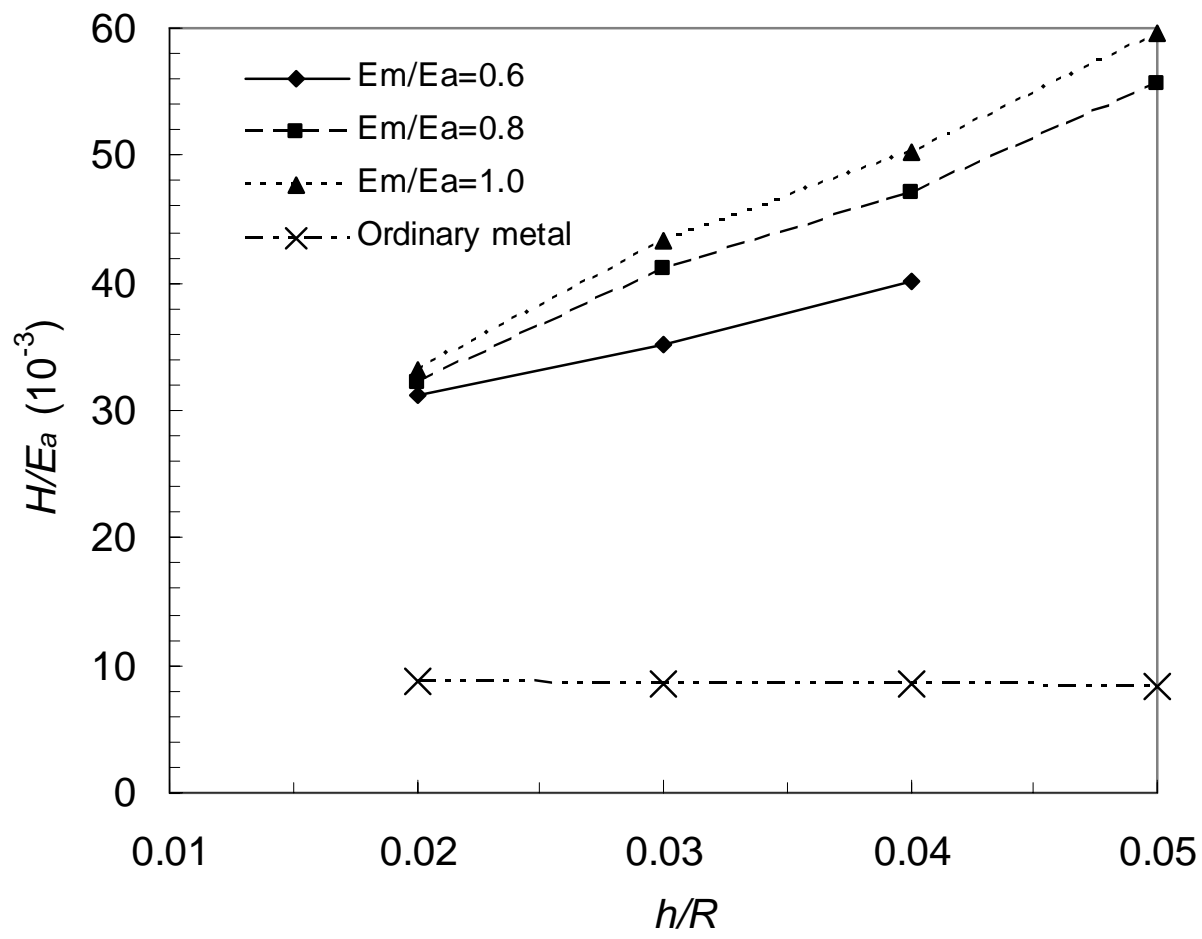

Fig. 8. Influence of the maximum indentation depth $h_{m} / R$ on the normalized hardness $H / E_{a}$ for different ratios of $E_{m} / E_{a}$ with $\varepsilon^{t r}=4 \%$ and $\sigma_{f} / E_{a}=0.012$, compared with the results from an ordinary elastic-perfectly plastic metal with $\sigma_{Y}=600 \mathrm{MPa}$ and $E=200$ GPa. 


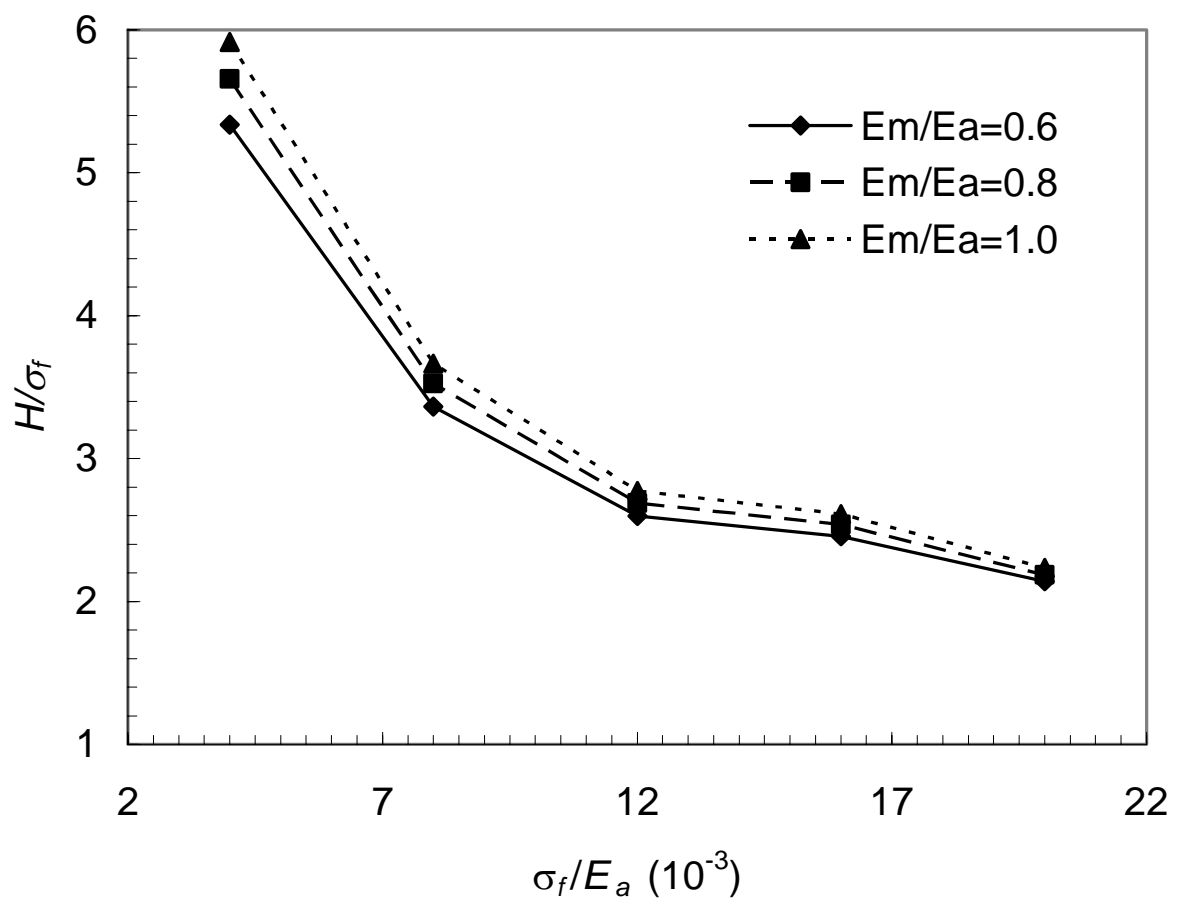

Fig. 9(a) 


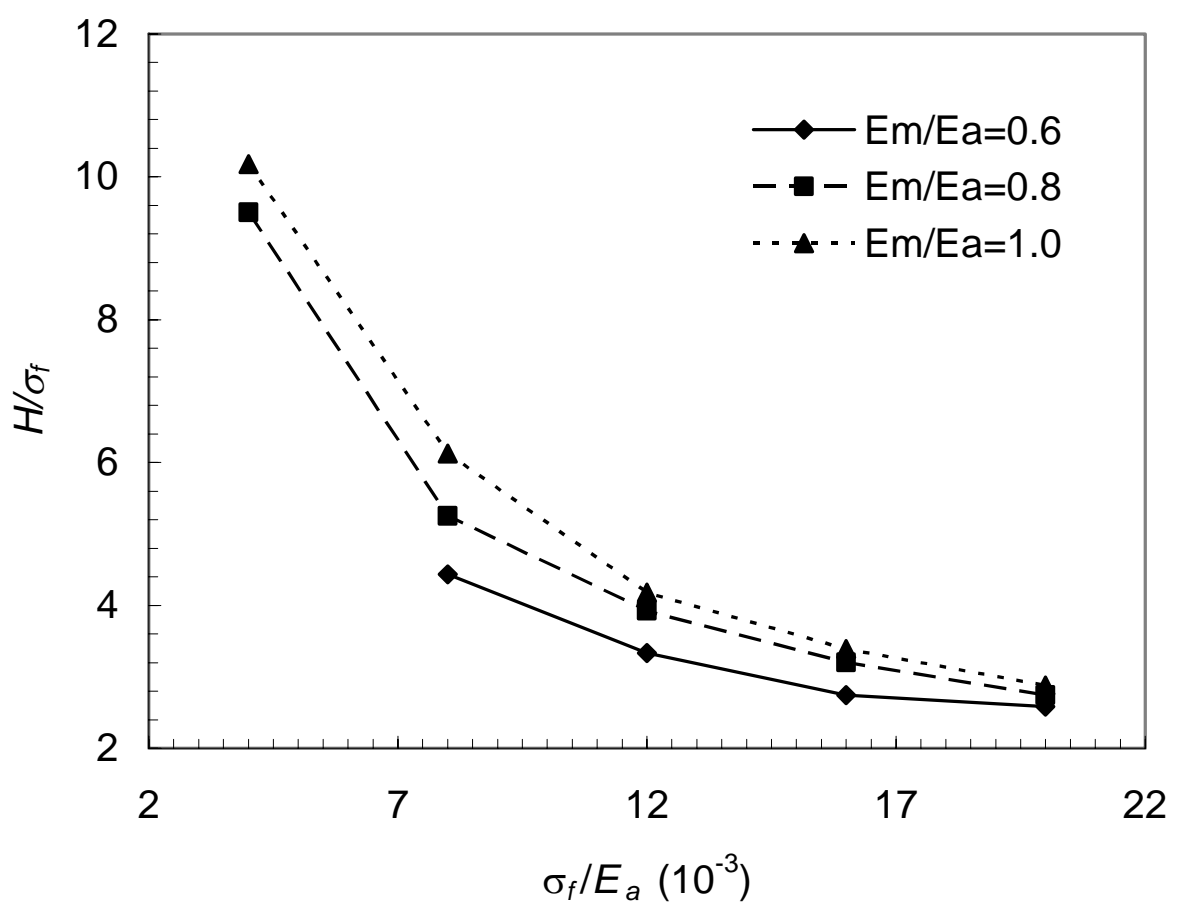

Fig. 9(b)

Fig. 9. Relationship between the ratio of the hardness to the forward transformation stress $H / \sigma_{f}$ and the normalized forward transformation stress $\sigma_{f} / E_{a}$ with $\varepsilon^{t r}=4 \%$ and (a) $h_{m} / R=0.02 ;$ (b) $h_{m} / R=0.04$ 


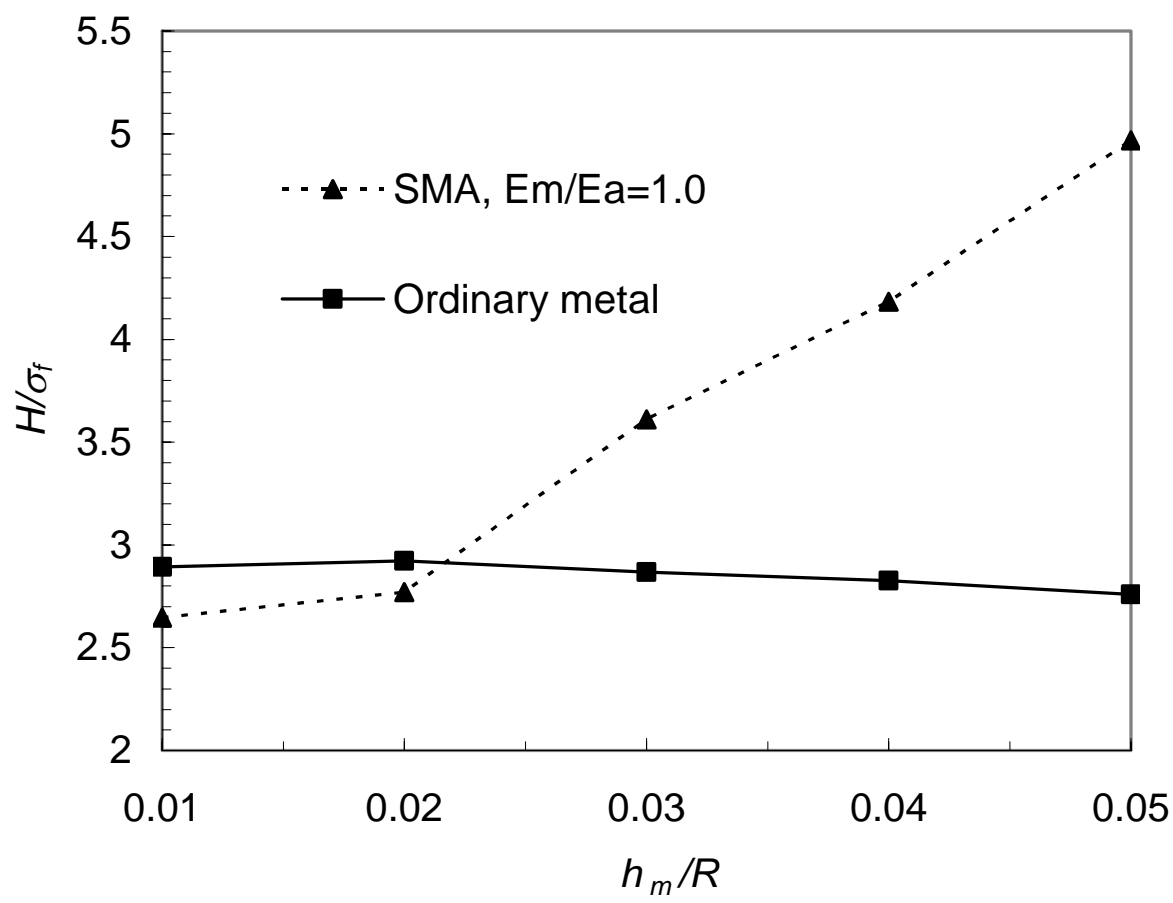

Fig. 10. Relationship between the ratio of the hardness to the forward transformation stress $H / \sigma_{f}$ and the normalized indentation depth $h / R$ for $\sigma_{f}=600 M P a$ and $\varepsilon^{t r}=4 \%$, compared with an ordinary elastic-perfectly plastic material with $\sigma_{Y}=600 \mathrm{MPa}$ and $E=$ $200 \mathrm{GPa}$.

Submitted to Materials Science and Engineering A. 